\title{
Simulating the dynamics of binary black holes in nuclear gaseous discs
}

\author{
Massimo Dotti ${ }^{1}$, Monica Colpi ${ }^{2}$, Francesco Haardt ${ }^{1}$ and \\ Lucio Mayer ${ }^{3,4}$ \\ ${ }^{1}$ Dipartimento di Fisica e Matematica, Università dell'Insubria, 22100, Como, Italy \\ email: dotti@mib.infn.it \\ ${ }^{2}$ Dipertimento di Fisica G. Occhialini, Università di Milano Bicocca, 20126 Milano, Italy \\ ${ }^{3}$ Institute for Theoretical Physics, University of Zurich, CH-8057, Zurich, Switzerland \\ ${ }^{4}$ Institute of Astronomy, Department of Physics, ETH, Zurich, HC-8095 Zurich, Switzerland
}

\begin{abstract}
We study the pairing of massive black holes embedded in a massive circum-nuclear, rotationally supported disc, until they form a close binary. Using high resolution SPH simulations, we follow the black hole dynamics, and in particular the eccentricity evolution, as a function of the composition in stars and gas of the disc. Binary-disc interaction always leads to orbital decay and, in case of co-rotating black holes, to orbit circularization. We present also a higher resolution simulation performed using the particle-splitting technique showing that the binary orbital decay is efficient down to a separation of $\sim 0.1 \mathrm{pc}$, comparable to our new resolution limit. We detail the gaseous mass profile bound to each black hole. Double nuclear activity is expected to occur on an estimated timescale of $\lesssim 10$ Myrs.
\end{abstract}

Keywords. black hole physics, hydrodynamics, galaxies: evolution, galaxies: nuclei, galaxies: starburst

\section{Introduction}

Understanding the binary orbital evolution is very important to constrain the history of formation and assembly of massive black holes (MBHs), and for predicting the gravitational wave (GW) signal detectable by the Laser Interferometer Space Antenna (LISA). In Dotti, Colpi \& Haardt (2006) we have shown that the interaction between a MBH binary and a pure gaseous massive disc circularizes the binary orbit. In the same framework, we extend our analysis by varying the composition of the disc, transforming a fraction of gaseous particles in stars.

\section{Results}

We consider the case of two MBHs in the disc plane, the primary is addressed in the disc centre of mass, while the secondary is on an initially highly eccentric orbit, $\mathrm{co}^{-}$ rotating with the disc at a separation from the centre of mass of $50 \mathrm{pc}$. The mass of the primary is $4 \times 10^{6} \mathrm{M}_{\odot}$, and the MBHs mass ratio is $1,1 / 4$ or $1 / 10$. The disc mass is $10^{8} \mathrm{M}_{\odot}$, composed by stars and/or gas. The stellar fractions of the disc mass in the different simulations is $0,1 / 3,2 / 3$, and 1 (for full details see Dotti et al. 2007).

The main results of our suite of simulations are:

- the sinking time of the initially eccentric MBH does not depend significantly on the fraction of gas particles transformed in stars;

- relevant orbit circularization of the initially eccentric MBH occurs in every disc model. 
The last result is opposite to what we found for a counter-rotating $\mathrm{MBH}$, or for a $\mathrm{co}^{-}$ rotating $\mathrm{MBH}$ in a collisionless spherical environment, where simulations show an increase of eccentricity (Dotti et al. 2006).

\subsection{Higher resolution simulations}

We have performed a higher resolution simulation applying the particle splitting technique (Kitsionas \& Whitworth 2002). This procedure ensures a resolution of $0.1 \mathrm{pc}$ without a significant increase of the computational time, and prevents at the same time spurious contamination between low and high resolution regions. The MBH binary does not stall (see also Escala et al. 2005), but rather reaches a separation of $0.1 \mathrm{pc}$, comparable to the new resolution limit.

The high resolution achieved in our simulation allows us to study the structure of gas particles bound to each individual MBH. We have found that during the orbital evolution, the gravitational attraction of each $\mathrm{MBH}$ on the environment is such that a mass (in gas) of $\approx 50 \%$ of the $\mathrm{MBH}$ mass is conveyed inside the $\mathrm{MBH}$ sphere of influence and that $\approx 2 \%$ of the $\mathrm{MBH}$ mass is deeply bound to each single $\mathrm{MBH}$. From the angular momentum profile of the particles bound to the MBHs, we can infer that the size of the geometrically thin accretion disc around each $\mathrm{MBHs}$ will be $\sim 0.01 \mathrm{pc}$, in presence of efficient cooling. We suggest that an active Eddington-limited accretion phase may set in, for a time $\lesssim 1$ Myrs around both MBHs.

\section{Conclusions}

In this proceeding we have shown that the dynamical friction against the gaseous and/or stellar background can drive the inspiral of $\mathrm{MBH}$ pairs inside massive nuclear discs. A MBH binary forms at a separation $\sim 5$ pc. The gas and stellar distribution around the binary is such that dynamical friction keeps acting on each $\mathrm{MBH}$ down to a separation of $\approx 0.1 \mathrm{pc}$, reducing the eccentricity to a value consistent with zero.

Given the mass profile of the gas bound around each $\mathrm{MBH}$, we speculate that two accretion discs of scale radius of $\sim 0.01 \mathrm{pc}$ may form during the last phases of the binary dynamical evolution, and that a double nuclear activity may be present for a time-scale of $\lesssim 1$ Myr.

\section{References}

Dotti, M., Colpi, M., \& Haardt, F. 2006, MNRAS 367, 103

Dotti, M., Colpi, M., Haardt, F., \& Mayer, L. 2007, MNRAS 379, 956

Escala, A., Larson, R. B., Coppi, P. S., \& Maradones, D. 2005, ApJ 630, 152

Kitsionas, S. \& Whitworth, S. 2002, MNRAS 330, 129 